\title{
Metal-organic framework glass anode with an exceptional cycling-induced capacity enhancement for lithium ion batteries
}

Chengwei Gao, Zhenjing Jiang, Peixing Wang, Lars Rosgaard Jensen, Yanfei Zhang*, Yuanzheng Yue*

C. Gao, Z. Jiang, P. Wang, Prof. Y. Zhang, Prof. Y. Yue

School of Materials Science and Engineering, Qilu University of Technology (Shandong Academy of Science), Jinan 250353, China

E-mail: zhang-yanfei@hotmail.com (Prof. Y. Zhang)

C. Gao, Prof. L.R. Jensen, Prof. Y. Yue

Department of Chemistry and Bioscience, Aalborg University, 9220 Aalborg, Denmark

E-mail: yy@bio.aau.dk (Prof. Y. Yue)

Prof. L.R. Jensen

Department of Materials and Production, Aalborg University, 9220 Aalborg, Denmark

Keywords: lithium ion batteries, metal organic frameworks, metal organic framework glass, capacity enhancement

\begin{abstract}
Metal organic frameworks (MOFs) hold great promise as high-energy anode materials for nextgeneration lithium ion batteries (LIBs) due to their tuneable pore structure and abundant reaction sites. However, since the pore structure of crystalline MOFs tends to collapse during lithium ion insertion and extraction, it has been a challenge to develop crystalline MOF-based anodes for high performance lithium ion batteries. Here we report a breakthrough in developing the high-performance MOF anodes. In detail, we have developed the first MOF glass anode, i.e., melt-quenched Cobalt-ZIF-62 glass anode with exceptional electrochemical performances. Compared with its crystalline counterpart, the Co-ZIF-62 glass anode exhibits significantly higher lithium storage capacity $\left(306 \mathrm{mAh} \mathrm{g}^{-1}\right.$ after 1000 cycles at the current density of $2 \mathrm{~A} \mathrm{~g}^{-}$ ${ }^{1}$ ), outstanding cycling stability and superior rate performance. Amazingly, the Li-ion storage capacity of the MOF glass anode continuously rises with increasing the number of chargedischarge cycles and even tripled after 1000 cycles. We revealed the possible origin of the unusual cycling-enhanced performances of the MOF glass anode. These superior performances make MOF glasses ideal candidates for anode materials for LIBs.
\end{abstract}




\section{Introduction}

Confronting the gradual depletion of fossil energy and the subsequent environmental degradation, it is the utmost urgency to develop advanced sustainable energy storage and conversion systems. To date, lithium ion batteries (LIBs), as one of the vital energy storage devices, still dominate the commercial markets. However, large-scale energy storage devices and hybrid electric vehicles demand breakthroughs in enhancing the power/energy density, safety and cycling life of the LIBs. In this context, extensive research are being conducted to seek new anode materials with superior lithium storage performance. ${ }^{[1-3]}$

Metal organic frameworks (MOFs), assembled by metal ions and organic ligands, are considered to be one of the most promising anode candidates for high-performance LIBs owing to their great porosity, abundant reaction sites and tunable structures. ${ }^{[4-6]}$ The polycrystalline MOF-177 was first applied as the anode material for LIBs, delivering a high initial irreversible capacity of $400 \mathrm{mAh} \mathrm{g}^{-1}$. However, the capacity rapidly decayed to $105 \mathrm{mAh} \mathrm{g}^{-1}$ after only two cycles. ${ }^{[7]}$ Although some MOFs have great potential to be the anode materials of LIBs, the poor cycling stability due to the total destruction of the framework is still a big challenge yet to be overcome. The structural collapse in most of MOFs anodes was found to be caused by the interactions between the metal-ligand coordination bonds in MOFs and lithium ions during cycling processes. ${ }^{[7]}$ Moreover, MOFs face another significant challenge of poor electronic conductivity for electrochemical applications. To overcome these challenges and to improve the electrochemical properties, pure MOFs are generally used as hosts of metal oxide nanoparticles to prepare composites, or as templates/precursors to synthesize MOFs derivatives such as porous carbons and carbon composites. ${ }^{[8-10]}$ However, these processes are usually realized at the expense of destroying the intrinsic organic-inorganic hybrid structures of MOFs. Therefore, it is critically important to ensure the structure integrity of MOFs for maintaining both the active surface area and the electronic conductivity in LIB anodes during operation. 
To this end, we have recently proposed the order/disorder engineering concept to enhance the electrochemical performances of Al-MOF/graphene composite anode for LIBs. ${ }^{[11]}$ Specifically, the partial disordering in the Al-MOF particles induced by the lithiation/delithiation process enabled efficient lithium storage and cycling stability enhancement owing to the reduced $\mathrm{Li}^{+}$ ion diffusion distance and the opened structure to buffer the mechanical stresses. Besides the order-disorder transition during the lithiation/delithiation process, the amorphization of polycrystalline MOFs can also be achieved via many other different routes, e.g., mechanosynthesis, ${ }^{[12]}$ mechanical milling, ${ }^{[13]}$ thermal ${ }^{[14]}$ and pressure treatment. ${ }^{[15]}$

In terms of the microstructure, the mechanically amorphized MOFs inherit some of the crystal characteristics, e.g., the short- and medium-range order, framework connectivity, and porosity, while both long-range disorder and defects can be induced during the mechanical milling process. ${ }^{[15]}$ In addition to mechanically amorphized MOFs, another class of disordered MOFs emerged six years ago, i.e., the so-called melt-quenched (MQ) zeolitic-imidazole framework (ZIF) glasses. ${ }^{[16]}$ Note that ZIFs are a sub-class of MOFs, and some of ZIFs can be meltquenched to glass state. ${ }^{[16,17]}$ Out of the ZIF glass systems studied so far, ZIF-62 is the one with the highest glass stability and glass forming ability. The microstructure of MQ-ZIF glasses are rather different from those of their counterparts: crystalline ZIFs and mechanically amorphized ZIFs, e.g., in short range disorder, defect concentration and pore structure. Very recently, scientists discovered the direct evidence for a high degree of short-range disorder in ZIF glasses using ultrahigh-field $\mathrm{Zn}^{67}$ solid-state nuclear magnetic resonance spectroscopy. ${ }^{[18]}$ The shortrange disorder arises from the lower rigidity and larger size of the tetrahedral units in comparison to inorganic network glasses that possess short-range order structure. These distinct features aroused our great curiosity about whether MQ-ZIF glasses could be utilized as superior anode materials in LIBs, since they could be beneficial to the lithium ion transfer kinetics. We attempt to find out which ZIF structure has the greatest potential to be used for fabricating high- 
performance LIB anode, i.e., among melt-quenched structure, mechanically amorphized structure and crystalline structure.

The standard ZIF-62 structure is constructed by the central metal nodes (Zinc) and organic ligands such as imidazolate (Im) and benzimidazolate (bIm), which is expressed by the chemical formula $\mathrm{Zn}\left[\operatorname{Im}_{1.75} \mathrm{bIm}_{0.25}\right]$. The nodes and the linkers are connected by coordination bonds that are much weaker than covalent bonds inside the linkers. According to literature, the crystalline structure and pore structure of Zn-ZIF can be modified by gradually substituting cobalt $(\mathrm{Co})$ for $\mathrm{Zn} .{ }^{[19]}$ When all $\mathrm{Zn}$ atoms are replaced by Co atoms, the standard Co-ZIF-62 is obtained, which has higher porosity and larger pores than Zn-ZIF-62. It has been found that CoZIF-62 can be melt-quenched to glass state despite its relatively low glass forming ability compared to Zn-ZIF-62 ${ }^{[20]}$. Moreover, it has been confirmed that the pores in Co-ZIF-62 crystal can be retained in its glass state upon quenching, and hence, Co-ZIF-62 glass is made an excellent material for gas separation and storage. ${ }^{[20,21]}$

By taking advantage of the nano porous structure of MQ Co-ZIF-62 glass, in this work we fabricated the LIB anode from this glass and characterized electrochemical performances. In parallel, we also prepared both the crystalline Co-ZIF-62 and the mechanically amorphized ZIF-62 (Co) anodes, which are noted as ZIF crystal and amorphous ZIF for simplicity and determined their electrochemical properties. Then we made a detailed comparison among the three types of anodes concerning their cycling performance and rate capability. Amazingly, the ZIF-62 glass anode exhibits excellent electrochemical performances, and in particular, the $200 \%$ enhancement of the capacity caused by 1000 discharging/charging cycles at a high current density of $2 \mathrm{~A} \mathrm{~g}^{-1}$. To the best of our knowledge, it is the first MOF glass anode for LIBs, which was produced and tested since the discovery of MOF glass family five years ago. ${ }^{[16]}$

\section{Fabrication and Material Characteristics}


Scheme 1 shows the schematic representation of the preparation of ZIF crystal, ZIF glass and amorphous ZIF for anode material in LIBs. The ZIF crystal was prepared by a solvothermal method with slight modifications. ${ }^{[22]}$ The ZIF glass was obtained by quenching the ZIF crystal melt to room temperature and the amorphous ZIF was produced by subjecting ZIF crystal to a high-energy ball milling process. As shown in Figure 1a, the powder X-ray diffraction (PXRD) pattern of the as-prepared ZIF crystal resembles that of the simulated pattern (CCDC No. 671070), confirming the successful preparation of the ZIF-62 (Co) crystal. ${ }^{[22]}$ After meltquenching and ball milling, all the Bragg diffraction peaks assigned to ZIF crystal disappear, indicating that the structure of ZIF crystal are transformed into the disordered and higher potential energy state. ${ }^{[16]}$ It should be mentioned that the structure of ZIF glass is twisted and disordered at all the length scales (i.e., short-, medium-, and long-ranges). In contrast, the shortand medium-range structure of amorphous ZIF is still not clear, but the long-range structure is disordered owing to the formation of a large concentration of the defects caused by mechanical impact. The subtle structural differences between ZIF glass and ZIF can be revealed by the differential scanning calorimetry (DSC). As shown in Figure 1b, during a DSC upscan at $10{ }^{\circ} \mathrm{C}$ $\min ^{-1}$, the as-prepared ZIF crystal undergoes solvent release that causes the endothermic peak at $270{ }^{\circ} \mathrm{C}$, and then melting event that causes the endothermic peak at around $435^{\circ} \mathrm{C}$. When the ZIF melt is cooled back to room temperature at $10{ }^{\circ} \mathrm{C} \mathrm{min}^{-1}$, the ZIF glass is obtained. During reheating of the glass at $10^{\circ} \mathrm{C} \mathrm{min}^{-1}$, the glass transition peak occurs, which is the typical feature of the melt-quenched glass. The onset temperature of the peak is defined as the standard glass transition temperature $\left(T_{\mathrm{g}}\right),{ }^{[23]}$ which is $325^{\circ} \mathrm{C}$ for the ZIF glass. It is seen in Figure $1 \mathrm{~b}$ that amorphous ZIF has the similar calorimetric responses to those of ZIF crystal in heating process, but the enthalpy and characteristic temperatures differ between the two samples. To evaluate the thermal stability, thermogravimetry (TG) curves of three samples were collected (Figure 1c). Significant mass losses are observed in TG curves from 100 to $300{ }^{\circ} \mathrm{C}$ for both the ZIF crystal and amorphous ZIF owing to the solvent release while nearly no mass loss is observed 
for ZIF glass. Moreover, the onset temperature of the mass loss in amorphous ZIF is lower than that in ZIF crystal. This implies that DMF is easier to be released from amorphous ZIF due to its broken framework during preparation than ZIF crystal. Figure 1d shows the Raman shifts of the three ZIF samples. It is seen that these samples exhibit similar Raman peak positions in the range of $200 \sim 1100 \mathrm{~cm}^{-1}$, and a sharp peak around $680 \mathrm{~cm}^{-1}$, implying that the organic ligands in ZIFs remain intact upon either melt-quenching or high-energy mechanic ball milling. In contrast, some differences in the Raman peak are observed in the range of $1100 \sim 1300 \mathrm{~cm}^{-1}$. In particular, the sharp peak at $1160 \mathrm{~cm}^{-1}$ of the ZIF crystal becomes considerably broader and weaker upon either mechanical amorphization or melt-quenching (see also Figure S1). This could be attributed to the distortion of the $\mathrm{C}-\mathrm{N}$ bonds during the mechanic amorphization and vitrification processes $^{[24-26]}$.

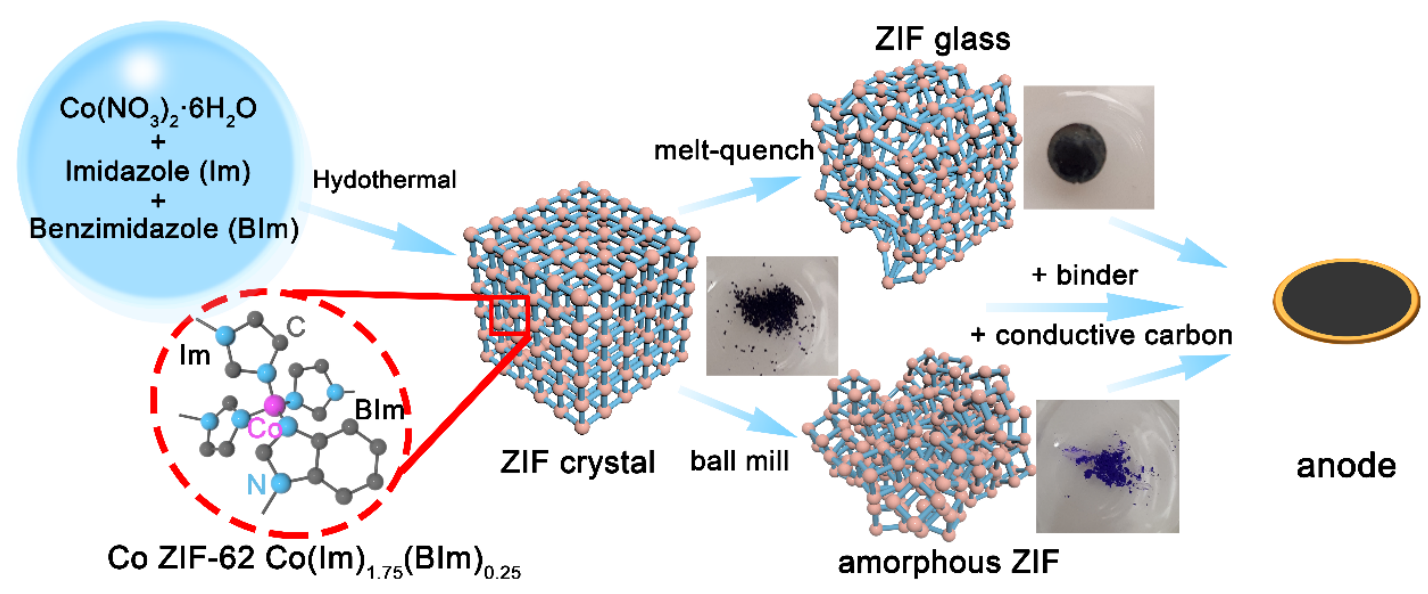

Scheme 1. Preparation of ZIF crystal (Co ZIF-62, Co(Im) $)_{1.75}(\mathrm{BIm})_{0.25}$ ), ZIF glass and amorphous ZIF by hydrothermal, melt-quenching and ball milling methods, respectively. Schematic representation the structure of Co ZIF-62 with hydrogen atoms omitted. Fabrication of anode: Mixing and pressing of the ZIF materials with carbon black and binder. 
(a)

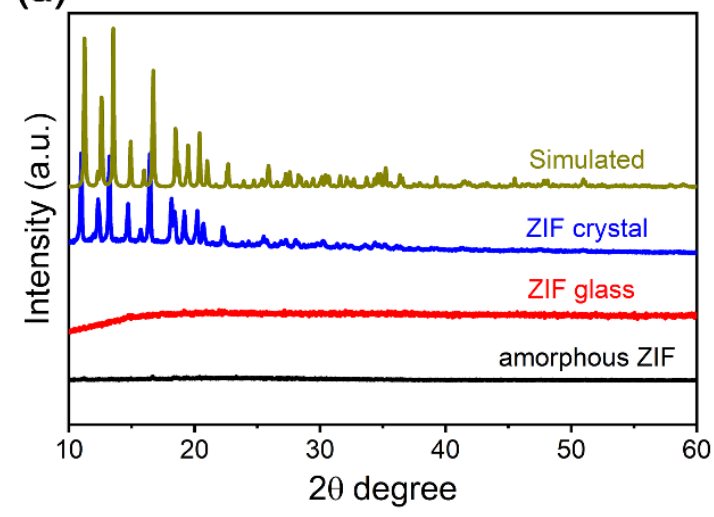

(c)

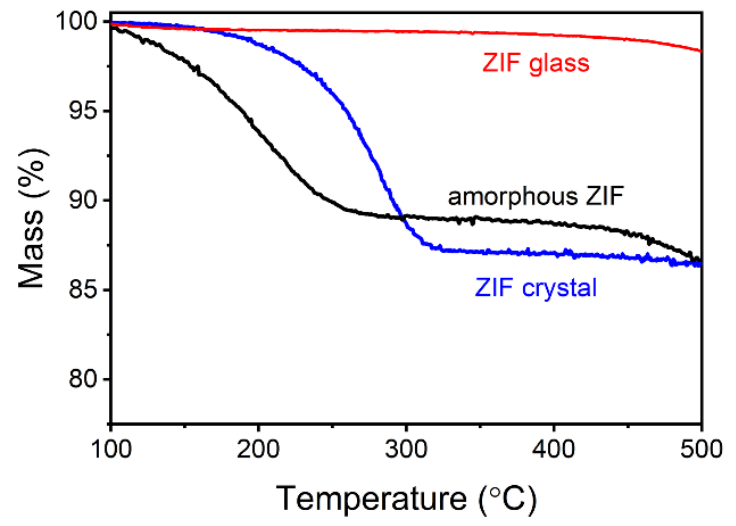

(b)

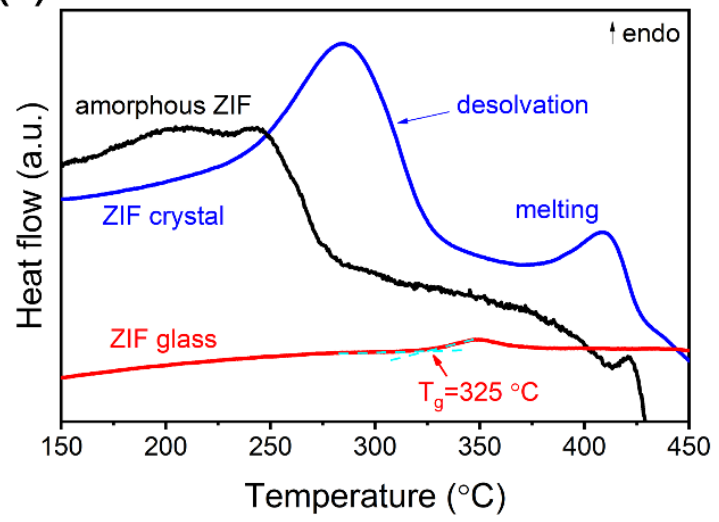

(d)

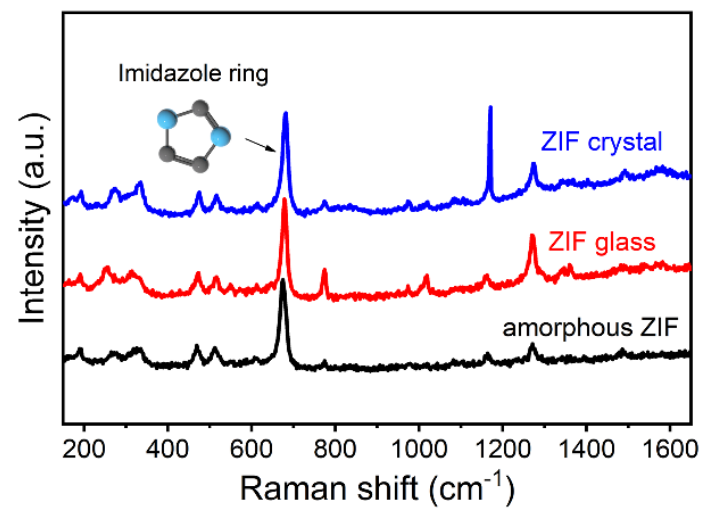

Figure 1. Structural characterizations of ZIF crystal, glass and amorphous solid. (a) X-ray diffraction patterns obtained from 10 to 60 degree $(2 \theta)$ showing the long-range order of ZIF crystal lost after the melt-quenching or ball milling process. (b) Heat flow and (c) Thermalgravimetric curves of the three ZIF samples during the DSC upscans at $10{ }^{\circ} \mathrm{C} \mathrm{min}^{-1}$ from 150 to $450^{\circ} \mathrm{C}$. (d) Raman spectra of all three ZIF samples sharing the same pattern.

The Fourier-transform infrared spectroscopy (FTIR) spectra of the three samples are shown in Figure S2. The peaks at $1676 \mathrm{~cm}^{-1}$ for both ZIF crystal and amorphous ZIF are associated with the vibrational mode of the carbonyl groups of solvent molecules (DMF) trapped in pores of ZIFs. In contrast, the $1676 \mathrm{~cm}^{-1}$ peak of ZIF glass is much weaker, and this can be ascribed to the fact that the DMF molecules escape from the sample during the prior heating process for glass formation. This agrees well with the results of TG curves (Figure 1c). In addition, the peaks at $1465,1235,1082,668 \mathrm{~cm}^{-1}$ could be observed in all three spectra, corresponding to C- 
$\mathrm{N}$ stretching, $\mathrm{C}-\mathrm{H}$ in-plane-bending, $\mathrm{C}-\mathrm{H}$ out-of-plane-bending and ring deformation out-ofplane-bending, respectively, ${ }^{[27]}$ which originate from the organic ligands of ZIF-62. In short, both the Raman and FTIR spectra confirms that the integrity of organic ligands is preserved in the three samples after the melt-quenching or ball milling process.

(a)

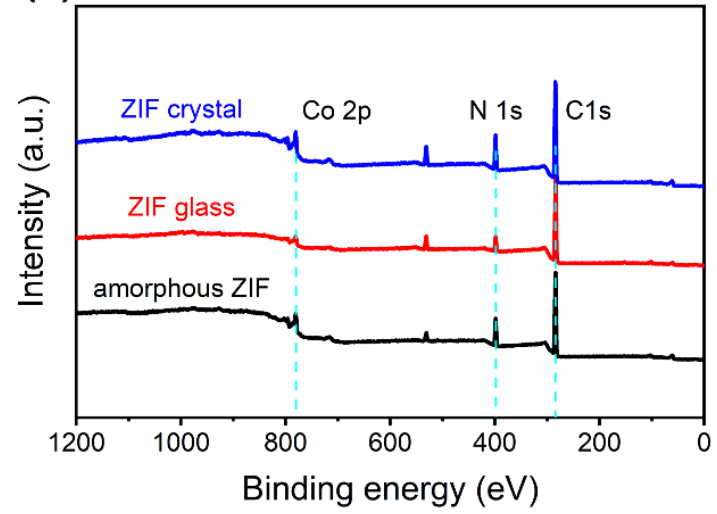

(c)

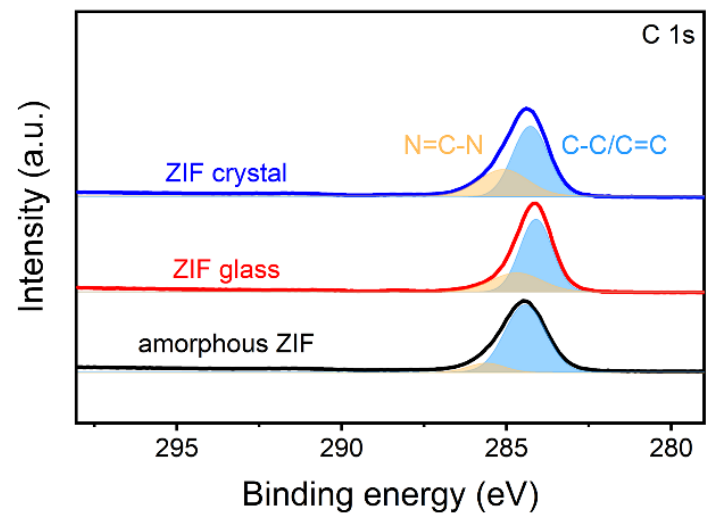

(b)

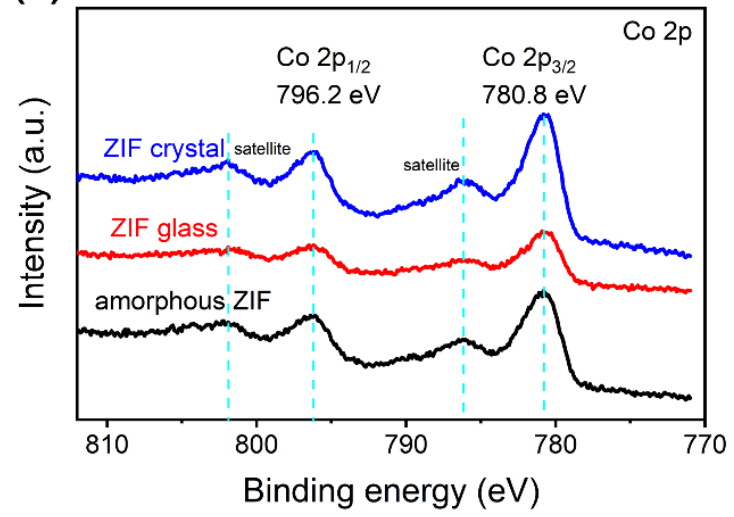

(d)

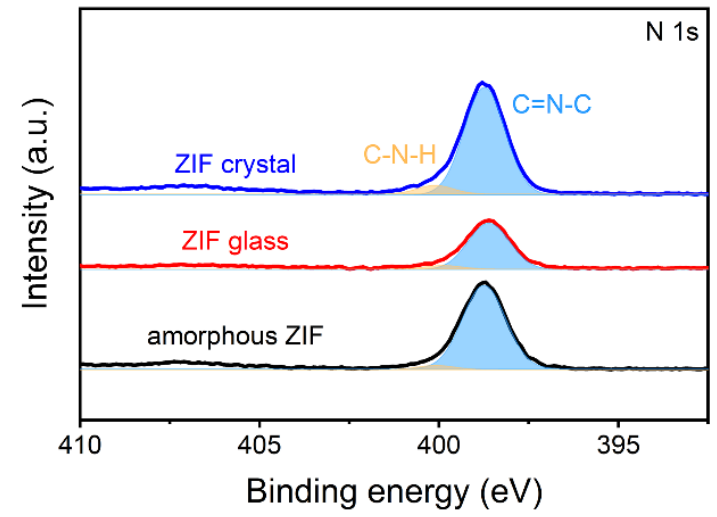

Figure 2. Chemical composition of ZIF crystal, ZIF glass and amorphous ZIF. (a) XPS survey spectra, high resolution spectra of (b) Co $2 p$, (c) C 1s, and (d) N 1s, demonstrating that these three samples consist of same composition.

X-ray photoelectron spectroscopy (XPS) spectra were collected to reveal the surface chemistry of the three ZIF samples. From the survey spectra in Figure 2a, the elements $(\mathrm{Co}, \mathrm{C}$ and $\mathrm{N})$ involved in the three samples can be detected. Figure $\mathbf{2 b}$ shows the core level spectra of Co $2 p$, in which the Co $2 \mathrm{p}_{1 / 2}$ and Co $2 \mathrm{p}_{3 / 2}$ peaks locate at 796.2 and $780.8 \mathrm{eV}$ with two satellite peaks at 801.9 and $786.2 \mathrm{eV}$, respectively. The three samples exhibit the same peaks of Co $2 \mathrm{p}$, 
implying that the majority of Co exists as $\mathrm{Co}^{2+}$, which remains unaffected during the meltquenching or ball milling process ${ }^{[28]}$. In Figure 2c, the peak of $\mathrm{C} 1 \mathrm{~s}$ for each sample can be deconvoluted into two peaks assigned to $\mathrm{C}-\mathrm{C} / \mathrm{C}=\mathrm{C}$ at $284.2 \mathrm{eV}$ and $\mathrm{N}=\mathrm{C}-\mathrm{N}$ at $286 \mathrm{eV}$, respectively ${ }^{[29]}$. In the N 1s core level XPS spectrum (Figure 2d), the peaks at 398.7 and 399.5 $\mathrm{eV}$ arises from the bonds of $\mathrm{C}=\mathrm{N}-\mathrm{C}$ and $\mathrm{C}-\mathrm{N}-\mathrm{H}$, respectively ${ }^{[29]}$. It is evident that the three samples present almost identical C 1s and N 1s spectra, suggesting that both the melt-quenching and ball milling treatments have little impact on the imidazole and benzimidazole ligands in ZIFs. As shown in Figure S3, the average pore sizes of ZIF crystal, ZIF glass and amorphous ZIF from $\mathrm{N}_{2}$ adsorption-desorption isotherms are found to be $3.4,4.3$ and $3.8 \mathrm{~nm}$, respectively. From the above-presented results, we can infer that both the melt-quenching and the ball milling processes did not affect the chemical composition of Co-ZIF-62 but made its microstructure highly disordered. Next, we will investigate how the disorder of the ZIF structure impacts on the electrochemical performances of the ZIF based anode for LIBs.

\section{Electrochemical Performances}

The electrochemical performances of the anodes made from the ZIF crystal, ZIF glass and amorphous ZIF, respectively, are characterized by galvanostatic charge/discharge cycling, cyclic voltammetry, and electrochemical impedance spectroscopy. As shown in Figure 3a, S4a and $\mathbf{S 4 b}$, the three anodes display similar charge-discharge profiles, in which the first cycle curve has a discharge-charge plateau at 0.14 and $1.2 \mathrm{~V}$, respectively. In the following cycles, the discharge-charge plateau of the three samples shift to 0.46 and $1.2 \mathrm{~V}$, respectively, confirming the similar lithiation/delithiation processes of the three ZIFs anodes. 
(a)

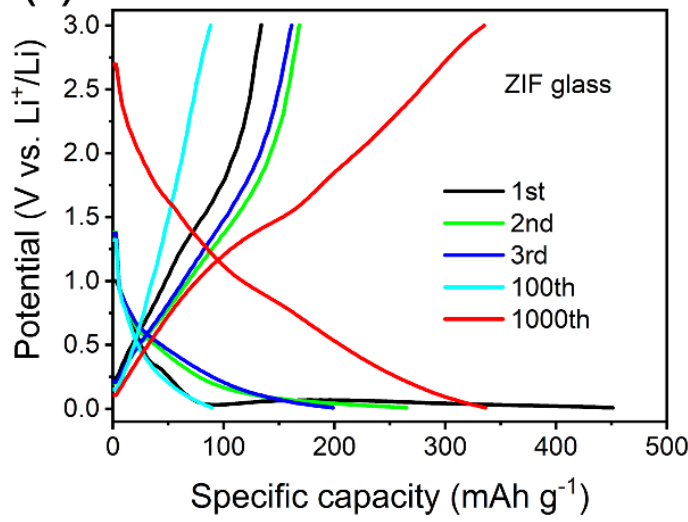

(c)

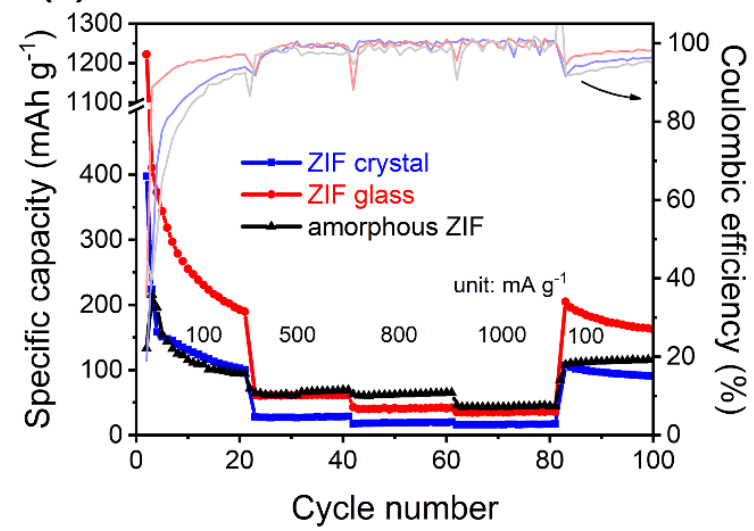

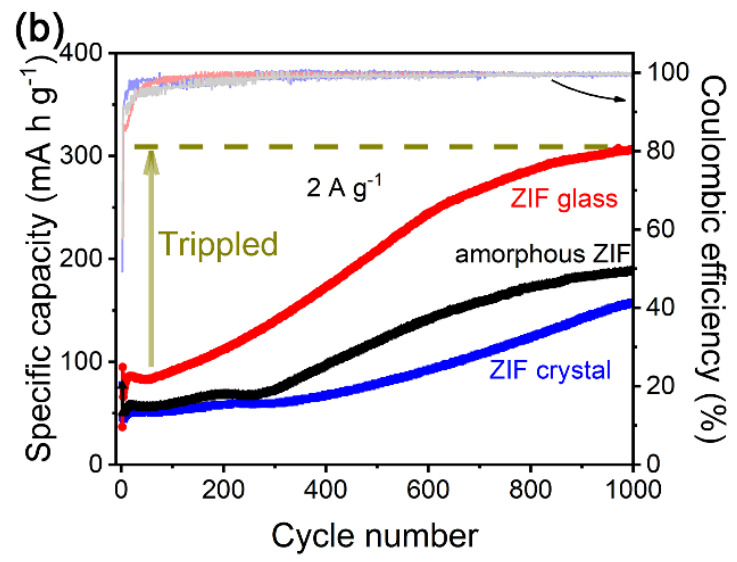

(d)

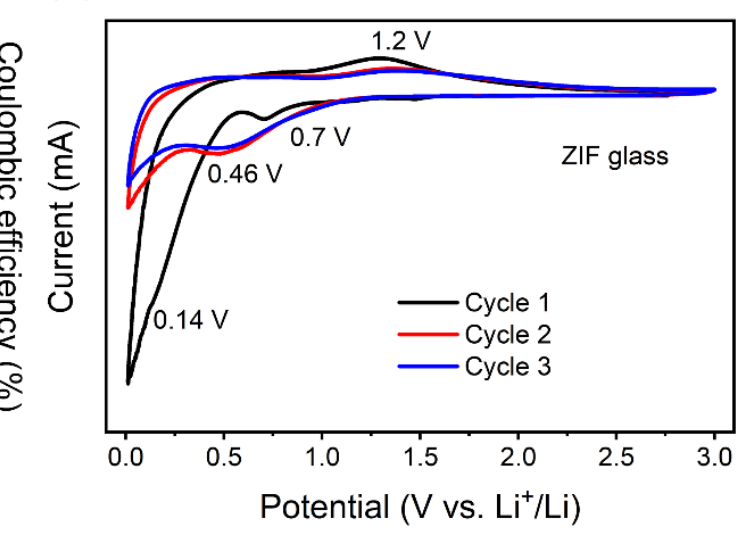

Figure 3. Electrochemical properties of ZIF crystal, ZIF glass and amorphous ZIF. (a) Galvanostatic charge-discharge profiles of ZIF glass for different cycles at the current density of $1 \mathrm{~A} \mathrm{~g}^{-1}$. (b) The cycling performance of ZIF crystal, ZIF glass and amorphous ZIF at $2 \mathrm{~A} \mathrm{~g}^{-}$ ${ }^{1}$ for 1000 cycles. (c) Rate performance of the three samples at current densities of 100, 500, 800, 1000 and $100 \mathrm{~mA} \mathrm{~g}^{-1}$ for 20 cycles. (d) Cyclic Voltammetry (CV) curves of ZIF glass within the range from 0.01 to $3.0 \mathrm{~V}$ at a scan rate of $0.1 \mathrm{mV} \mathrm{s}^{-1}$.

Highly stable cycling performance of electrodes plays an important role in the practical application of rechargeable batteries. In this regard, the cycling performances of the three above-mentioned anodes are evaluated at $2 \mathrm{~A} \mathrm{~g} \mathrm{~g}^{-1}$ (Figure 3b), as well as at $0.1,0.5$ and $1 \mathrm{~A} \mathrm{~g} \mathrm{~g}^{-1}$ (Figure S4c, S4d and S4e). At $0.5 \mathrm{~A} \mathrm{~g}^{-1}$, the discharge capacity $\left(670 \mathrm{~mA} \mathrm{~h} \mathrm{~g}^{-1}\right)$ of the ZIF glass is significantly higher than those of other two samples $\left(220 \mathrm{~mA} \mathrm{~h} \mathrm{~g}^{-1}\right.$ for ZIF crystal and 125 $\mathrm{mA} \mathrm{h} \mathrm{g}^{-1}$ for amorphous ZIF) at the first cycle. However, the capacity of the ZIF glass decays 
to about $200 \mathrm{~mA} \mathrm{~h} \mathrm{~g}^{-1}$ after 20 cycles and remains unchanged till 100 cycles. This capacity is higher than those of both the ZIF crystal (149 $\left.\mathrm{mA} \mathrm{h} \mathrm{g}^{-1}\right)$ and amorphous ZIF (125 mA h g $\left.{ }^{-1}\right)$ (also see Figure S4d). In Fig. 3b, we can see an unusual phenomenon, that is, the three samples display a continuous increase of the capacity during 1000 cycles at $2 \mathrm{~A} \mathrm{~g}^{-1}$. Specifically, the capacities of ZIF crystal, ZIF glass and amorphous ZIF have reached 157, 188 and $306 \mathrm{~mA} \mathrm{~h}$ $\mathrm{g}^{-1}$, respectively, at $1000^{\text {th }}$ cycle at $2 \mathrm{~A} \mathrm{~g}^{-1}$. It is clearly seen that, compared to ZIF crystal and amorphous ZIF, the ZIF glass shows a significantly greater enhancement in capacity after 1000 cycles, i.e., its capacity is tripled relative to the initial value of $95 \mathrm{mAh} \mathrm{g}^{-1}$. Under the same condition, both ZIF crystal and amorphous ZIF exhibit the capacity enhancement as well, but to a lesser extent compared with that of ZIF glass anode (Figure S4f). When comparing the cycling curves between the amorphous ZIF and ZIF crystal, it is evident that the former shows slightly higher capacity than the latter. As shown in Figure 3c, the rate capacities of the three samples are determined at the current densities of $100,500,800$ and1000 $\mathrm{mA} \mathrm{g}^{-1}$, at each of which 20 cycles of discharging/charging are conducted. It is clearly seen that the capacity drops with increasing the current density. Remarkably, when the current density is switched back to $100 \mathrm{~mA} \mathrm{~g}^{-1}$, the capacities fully recover to the values corresponding to this current density for all the three samples.

To further evaluate the electrochemical performances of the three ZIF anodes, cyclic voltammetry $(\mathrm{CV})$ measurements during the rage from 0.01 to $3 \mathrm{~V}$ at a scan rates of $0.1 \mathrm{mV} \mathrm{s}^{-}$ ${ }^{1}$ were conducted. As shown in Figure 3d, the CV curves of ZIF glass present the reduction peaks at 0.7 and $0.14 \mathrm{~V}$ in the first cathodic scan, and these peaks might originate from insertion of $\mathrm{Li}^{+}$ions into ZIF and formation of solid electrolyte interface film (SEI) ${ }^{[30]}$. The peak at 1.2 $\mathrm{V}$ in the anodic scan is ascribed to the extraction of $\mathrm{Li}^{+}$ions from ZIF glass. In the subsequent two cycles, one couple of redox peaks $(0.46 / 1.2 \mathrm{~V})$ remain almost unchanged, corresponding to the reversible insertion/extraction of $\mathrm{Li}^{+}$ions into/from ZIF glass. As displayed in Figure 3d, S5a and S5b, the redox peaks of the three samples occur at almost the same potential. This 
further confirms that the three samples undergo an identical lithium storage mechanism despite their structural difference.

(a)

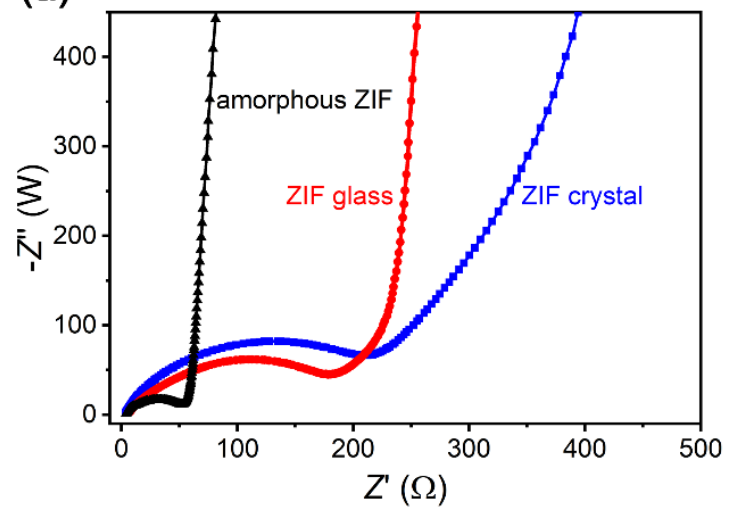

(c)

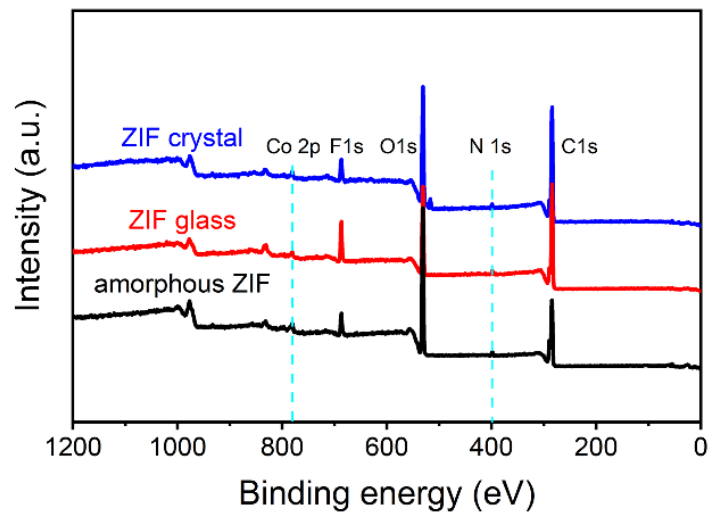

(b)

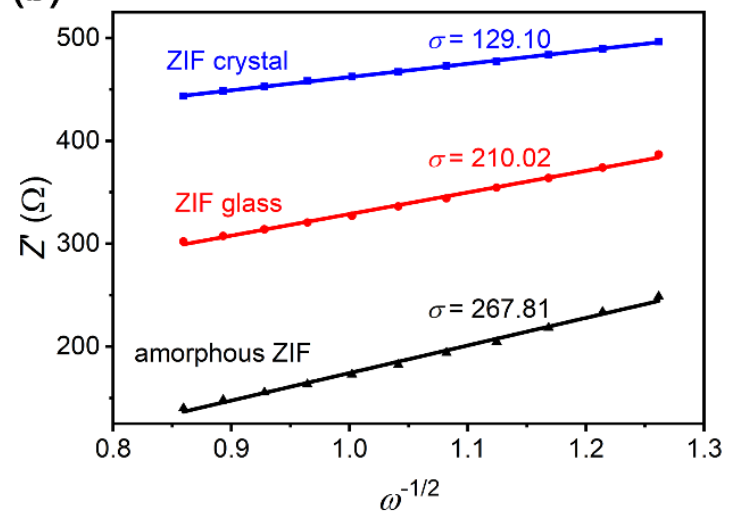

(d)

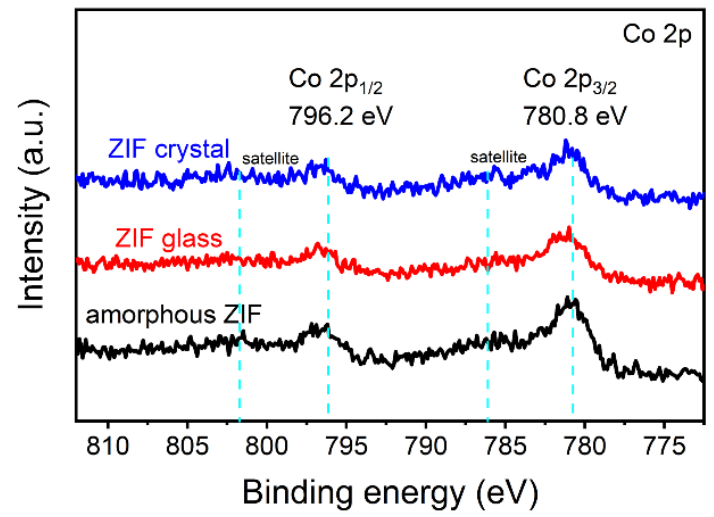

Figure 4. Impedance analysis, structural characterization of ZIF crystal, ZIF glass and amorphous ZIF. (a) Nyquist plots obtained from electrochemical impedance spectra within frequency range from 0.01 to $10 \mathrm{kHz}$ with an amplitude of $10 \mathrm{mV}$. (b) $Z^{\prime}$ vs. $\omega^{-1 / 2}$ plots in the low frequency region obtained from Nyquist plots. (c) XPS survey spectra and (d) the highresolution spectra of Co 2p of ZIF crystal, ZIF glass and amorphous ZIF after 1000 chargedischarge cycles, respectively.

To observe the difference in electrical resistance among the three ZIF anodes, we conducted electrochemical impedance spectroscopy measurement as shown in Figure 4a. It is seen that the Nyquist plots of the three anodes consist of a depressed semicircle in high frequency range 
and a straight line at low frequency end. The equivalent circuit model of the experimental curves is shown in Figure S6. The $R_{\mathrm{b}}$ (bulk resistance of the cell) of ZIF crystal, ZIF glass and amorphous ZIF are found to be 2.68, 4.95 and 3.57 $\Omega$, respectively (Table S1). The $R_{\mathrm{ct}}$ (charge transfer resistance) of the amorphous ZIF is the smallest (52 $\Omega$ ) among the three samples, indicating the highest electrical conductivity. The low-frequency sloping line is ascribed to the Warburg impedance, which is associated with the diffusion of $\mathrm{Li}^{+}$ions in the electrode material. Thus, the apparent $\mathrm{Li}^{+}$ion diffusion coefficient $D$ of these three samples are calculated from the incline line in the Warburg region using equation ${ }^{[31]}$ :

$D=R^{2} T^{2} / 2 A^{2} n^{4} F^{4} C^{2} \sigma^{2}$

where $R$ is the gas constant, $T$ is the absolute room temperature, $A$ is the surface area of the electrode $\left(\sim 1.13 \mathrm{~cm}^{2}\right), n$ is the number of electron transferred during oxidation, $F$ is the Faraday constant, $C$ is the $\mathrm{Li}^{+}$concentration, and $\sigma$ is the Warburg factor associated with $Z_{\text {re }}\left(Z_{\text {re }} \propto \sigma \omega^{-1 / 2}\right)$. $\sigma$ can be determined from the linear relation between $Z_{\text {re }}$ and the reciprocal square root of the angular frequency $\omega$ (Figure $\mathbf{4 b}$ ). By introducing $\sigma$ into Equation (1), we can calculate the apparent $\mathrm{Li}^{+}$diffusion coefficient $D$ since the temperature and other constants are known. Thus, the $D$ values of ZIF crystal, ZIF glass and amorphous ZIF are found to be $2.82 \times 10^{-14}, 1.06 \times$ $10^{-14}$ and $6.54 \times 10^{-15} \mathrm{~cm}^{2} \mathrm{~s}^{-1}$, respectively.

To confirm that the electrochemical performance differences among the three samples arise from their structural difference, we also carried out the XPS measurements of the anodes that have been subjected to 1000 charge-discharge cycles (Figure 4c). It is clearly seen that F and $\mathrm{O}$ are present in all three samples, which originate from the SEI film. To find out whether the cycling process affects the valence state of cobalt, we highlight the Co $2 p$ spectrum in Figure 4d, which exhibits two peaks at $796.2 \mathrm{eV}\left(\mathrm{Co} 2 \mathrm{p}_{1 / 2}\right)$ and $780.8 \mathrm{eV}\left(\mathrm{Co} 2 \mathrm{p}_{3 / 2}\right)$ and two satellite peaks, indicating the existence of $\mathrm{Co}^{2+}$. This suggests that the valence state of $\mathrm{Co}$ is not influenced by 1000 charge-discharge cycles for all the three samples. In addition, the highresolution spectra of C 1s and $\mathrm{N}$ 1s also remain unchanged upon cycling as evidenced in Figure 
2 and S7. The $\mathrm{CO}_{3}{ }^{2-}$ peaks for the cycled samples originate from SEI layer (Figure S7). All the XPS results suggest that both the metal nodes and organic ligands in all ZIF samples remain unaltered during the charge-discharge process. To study the structure evolution during cycles, the ex-situ XRD patterns were also collected (Figure S8). It is striking that ZIF crystal lose its long-range order by the order-disorder transition ${ }^{[11]}$. In contrast, both ZIF glass and amorphous ZIF maintain their original disordered structure after cycling, as no diffraction peaks appear.

The extraordinary and unique cycling-induced enhancement of capacity in the glass anode (Figure 3b) could be associated with the disordered open network structure that is frozen-in during the melt-quenching process. In detail, such network structure possesses three striking features as follows. First, it is disordered at all the length scales such as short-range $(<0.8 \mathrm{~nm})$, medium-range $(8-20 \mathrm{~nm})$ and long-range $(>20 \mathrm{~nm})^{[16,18]}$, in contrast to other types of network glasses (e.g., oxide glasses). Second, the connection between metal nodes and ligands is established by the coordination bonds that are much weaker than those in the covalent and ionic bonds in inorganic glasses. Third, ZIF glass structure is also in highly metastable state with higher potential energy compared to ZIF crystal. These three features make ZIF glass more easily affected by $\mathrm{Li}^{+}$ion insertion and extraction under high current density, so that the number and size of the transfer channels for $\mathrm{Li}^{+}$ions would increase with cycling, and hence the capacity would be enhanced.

As mentioned early, the capacity of the ZIF crystal is also gradually enhanced with increasing the cycling number. The reason of this enhancement is the same as part of the reasons for ZIF glass, i.e., the weak coordination bonds between cobalt nodes and nitrogen atoms in the $\mathrm{Im} / \mathrm{bIm}$ ligands. During cycling, some of these bonds may easily be broken by $\mathrm{Li}^{+}$ion insertion/extraction, and thus the tetrahedral $\mathrm{Co}(\mathrm{Im} / \mathrm{bIm})_{4}$ network is partially depolymerized. This leads to the formation of larger channels (compared to the original ones), and hence, an increasing amount of $\mathrm{Li}^{+}$ions can be stored in ZIF crystal. However, due to its more stable structure, both the degree of the network depolymerisation and the structural defect 
concentrations are much lower than ZIF glass and amorphous ZIF, and therefore its capacity enhancement during cycling is less than that of the latter ones. Different from ZIF crystal, amorphous ZIF contains numerous structural defects such as broken bonds, unsaturated coordination for both nodes and ligands and vacancies, which are induced by mechanical impact during the ball milling process. These defects act as active sites for $\mathrm{Li}^{+}$ion storage and transfer. These defects also weaken the ZIF network, so that the $\mathrm{Li}^{+}$insertion/extraction can depolymerize the network, and hence, new channels are created. Therefore, the capacity of the amorphous ZIF anode is enhanced during cycling.

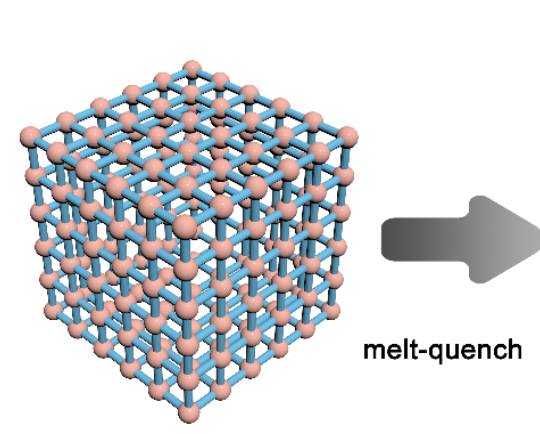

ZIF crystal

EI ZIF

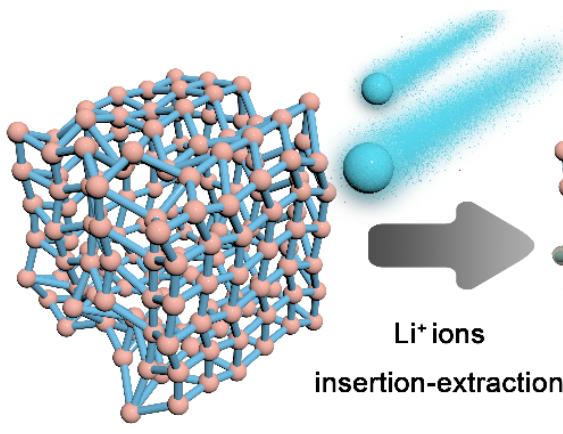

ZIF glass

$\mathrm{Li}^{+}$ions

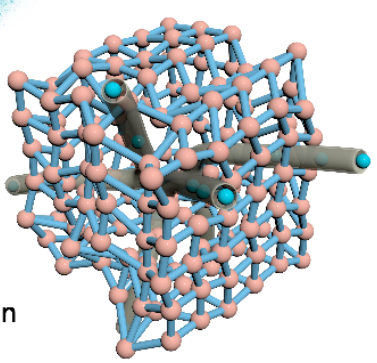

ZIF galss after cycling

$\mathrm{Li}^{+}$ions channels

Scheme 2. The schematic representation of the structural origin of the capacity enhancement in ZIF glass anode.

Based on the above discussions, the mechanism of the cycling-induced capacity enhancement in ZIF glass anode may be schematically illustrated in Scheme 2. Upon heating, ZIF crystal is melted, and thereby its network structure becomes disordered at all length scales (short-, medium- and long-range $)^{[16,18]}$. Upon quenching, the disordered structure is frozen-in in the glass state with higher potential energy (compared with crystal state). Once the $\mathrm{Li}^{+}$ions insert into the network structure, they will affect and disrupt the coordination bonds, and hence, generating new channels for $\mathrm{Li}^{+}$ion diffusion and storage. Consequently, the capacity of the glass anode substantially increases with cycling. Moreover, the short-range disorder in ZIF 
glass structure enables shortening the diffusion distance of $\mathrm{Li}^{+}$ions, benefiting the ionic transfer kinetics. In addition, the high-energy state of ZIF glass makes the network structure easily be distorted and depolymerized by insertion/extraction of $\mathrm{Li}^{+}$ions during charge-discharge cycles, and this favours the formation of new channels for $\mathrm{Li}^{+}$ion diffusion and storage. Thus, this type of ZIF glass (i.e. melt-quench Co ZIF-62 glass) exhibit great potential as a promising anode material for fabricating superior $\mathrm{Li}$ ion batteries.

\section{Conclusion}

We prepared the first ZIF glass anode for Li-ion batteries. This type of anode exhibits superior lithium storage performances such as higher capacity, greater rate capability and long-term cyclability, compared to its counterpart ZIF crystal. Astonishingly, ZIF glass exhibits a tripled increase of the specific capacity (up to $306 \mathrm{~mA} \mathrm{~h} \mathrm{~g}^{-1}$ ) after 1000 cycles at the current density of $2 \mathrm{~A} \mathrm{~g}^{-1}$. The outstanding electrochemical performances of ZIF glass have been ascribed to its unique structure characterized by a high degree of disorder at all scales and higher energy state nature. This encouraging discovery will allow us to develop the next generation of anodes for superior $\mathrm{Li}^{+}$ion batteries by using MOF glasses.

\section{Method}

Synthesis of ZIF crystal, ZIF glass and amorphous ZIF

Crystalline ZIF-62 (Co) was synthesized by a modified method based on previously literatures $^{[20,22,24,32,33]}$. In detail, imidazole (Im, $11.55 \mathrm{mmol}, 785.4 \mathrm{mg}$ ) and benzimidazole (BIm,

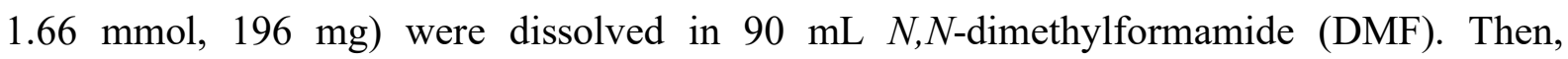
$\mathrm{Co}\left(\mathrm{NO}_{3}\right)_{2} \cdot 6 \mathrm{H}_{2} \mathrm{O}(4 \mathrm{mmol}, 1164 \mathrm{mg})$ was dissolved in above solution. After stirring for $0.5 \mathrm{~h}$, the solution was transferred into an oven and heated at $130{ }^{\circ} \mathrm{C}$ for 3 days. After cooling, the purple product was separated by centrifugation and dried under vacuum at $120{ }^{\circ} \mathrm{C}$ overnight. To obtain melt-quenched ZIF glass, the finely ground crystalline ZIF-62 was heated at $10{ }^{\circ} \mathrm{C}$ $\min ^{-1}$ to $450{ }^{\circ} \mathrm{C}$ and calcined at this temperature for 5 minutes followed by cooling naturally to room temperature. The whole process was purged with argon. The mechanical amorphous ZIF- 
62 was prepared by ball milling the crystalline sample at $500 \mathrm{rpm}$ for $10 \mathrm{~h}$. For simplicity, these samples (ZIF-62 (Co) crystal, melt-quenched ZIF and mechanical ball milling amorphous ZIF) are denoted as ZIF crystal, ZIF glass and amorphous ZIF, respectively.

\section{Material Characterizations}

Powder X-ray diffraction (XRD) measurements of the samples were performed on a PANalytical X-ray diffractometer with $\mathrm{Cu} \mathrm{K \alpha}(\lambda=1.5406 \AA)$ radiation during the $2 \theta$ range of $10-60^{\circ}$ with a step size of $0.013^{\circ}$. The thermal properties of samples were determined by the differential scanning calorimetry (DSC) (STA 404C Netzsch) in $\mathrm{N}_{2}$ atmosphere at a heating rate of $10{ }^{\circ} \mathrm{C} \mathrm{min}^{-1}$. Mass losses of samples with temperature were obtained simultaneously to the DSC scan by thermogravimetry (TG). The Raman spectroscopy measurements were conducted via Renishaw In-Via Raman microscopic with an $\operatorname{Ar}^{+}$laser $(\lambda=785 \mathrm{~nm})$ at $50 \times$ aperture. Fourier transform infrared (FTIR) spectra were recorded on a Bruker TENSOR II FTIR spectrometer with Platinum ATR Accessory at room temperature in the range of 400$4000 \mathrm{~cm}^{-1}$. X-ray photoelectron spectroscopy (XPS) was performed by using ESCALAB 250Xi spectrometer (ThermoFisher Scientific, USA) with non-monochromatic Al Ka X-ray $(1486.6 \mathrm{eV})$ at pass energy of $50 \mathrm{eV}$ to determine the surface properties and chemical compositions of the samples. $\mathrm{N}_{2}$ adsorption-desorption isotherms were collected at $77 \mathrm{~K}$ with an ASAP 2020 Accelerated Surface Area and Porosimetry System; Brunauer-Emmett-Teller (BET) and Barrett- Joyner-Halenda (BJH) analyses were performed to calculate the specific surface area, and the corresponding pore size distribution.

\section{Cell assembly and Electrochemical Characterizations}

The electrochemical performance of the as-prepared ZIF crystal, ZIF glass and amorphous ZIF were evaluated by coin-type cell CR2032 with metallic lithium foil (diameter of $16.0 \mathrm{~mm}$ ) as the counter electrode. The electrolyte consists of $1 \mathrm{M} \mathrm{LiPF}_{6}$ in ethylene carbonate (EC)/diethyl carbonate (DEC)/dimethyl carbonate (DMC) (1:1:1 vol\%) and a Celgard 2325 membrane (diameter of $19.0 \mathrm{~mm}$ ) functions as the separator. The working electrodes consist of the active 
powder materials $(70 \mathrm{wt} \%)$, conductive acetylene black $(20 \mathrm{wt} \%)$ as a conductor, and polyvinylidene difluoride (PVDF) (10 wt \%) as a binder. All the components were mixed well to form a slurry and pasted onto copper foil substrate. After being dried at $110{ }^{\circ} \mathrm{C}$ in a vacuum oven for $12 \mathrm{~h}$, the foil was cut into circular electrode with a diameter of $12 \mathrm{~mm}$. The loading mass of active materials is $1-2 \mathrm{mg}$, based on which the gravimetric capacity was calculated. The cells were assembled in an Argon-filled glovebox with both the moisture and the oxygen content below $1 \mathrm{ppm}$ and tested at $25{ }^{\circ} \mathrm{C}$. The galvanostatic charge/discharge tests of the cells were carried out on a Land battery test system (CT2001A). Cyclic voltammetry (CV) curves were recorded in the voltage range of $0.01-3 \mathrm{~V}$ at the scanning rate of $0.1 \mathrm{mV} \mathrm{s}^{-1}$ and EIS spectra were obtained in the frequency range of 0.1 to $100 \mathrm{kHz}$ on a $\mathrm{CHI} 760 \mathrm{e}$ electrochemical workstation with an amplitude of $5 \mathrm{mV}$.

\section{Supporting Information}

Supporting Information is available from the Wiley Online Library or from the author.

\section{Acknowledgements}

This work was financially supported by the China Scholarship Council (201707040085), the Taishan Young Scholarship Project of Shandong Province (tsqn202103098), the Shandong Provincial Natural Science Foundation (ZR2020ME025), the Colleges and Universities Twenty Terms Foundation of Jinan City (2019GXRC034) and Foundation from State Key Laboratory of Special Glass of China.

Received: ((will be filled in by the editorial staff))

Revised: ((will be filled in by the editorial staff))

\section{Conflict of Interest} Published online: ((will be filled in by the editorial staff))

The authors declare no conflict of interest.

\section{References}

[1] C. K. Chan, H. Peng, G. Liu, K. McIlwrath, X. F. Zhang, R. A. Huggins, Y. Cui, Nat. Nanotechnol. 2008, 3, 31 .

[2] J.-M. Tarascon, M. Armand, Nature 2001, 414, 359.

[3] G. N. Zhu, Y. G. Wang, Y. Y. Xia, Energy Environ. Sci. 2012, 5, 6652. 
[4] L. Wang, Y. Han, X. Feng, J. Zhou, P. Qi, B. Wang, Coord. Chem. Rev. 2016, 307, 361.

[5] L. Chen, W. Yang, J. Wang, C. Chen, M. Wei, Chem. Eur. J. 2018, DOI 10.1002/chem.201802629.

[6] S. Maiti, A. Pramanik, U. Manju, S. Mahanty, ACS Appl. Mater. Interfaces 2015, 7, 16357.

[7] X. Li, F. Cheng, S. Zhang, J. Chen, J. Power Sources 2006, 160, 542.

[8] H. Yue, Z. Shi, Q. Wang, Z. Cao, H. Dong, Y. Qiao, Y. Yin, S. Yang, ACS Appl. Mater. Interfaces 2014, 6, 17067.

[9] A. Banerjee, U. Singh, V. Aravindan, M. Srinivasan, S. Ogale, Nano Energy 2013, 2, 1158.

[10] W. Chaikittisilp, K. Ariga, Y. Yamauchi, J. Mater. Chem. A 2013, 1, 14.

[11] C. Gao, P. Wang, Z. Wang, S. K. Kær, Y. Zhang, Y. Yue, Nano Energy 2019, 65, 104032.

[12] T. D. Bennett, S. Cao, J. C. Tan, D. A. Keen, E. G. Bithell, P. J. Beldon, T. Friscic, A. K. Cheetham, J. Am. Chem. Soc. 2011, 133, 14546.

[13] Y. Ohara, A. Hinokimoto, W. Chen, T. Kitao, Y. Nishiyama, Y. Hong, S. Kitagawa, S. Horike, Chem. Commun. 2018, 54, 6859.

[14] T. D. Bennett, D. A. Keen, J. C. Tan, E. R. Barney, A. L. Goodwin, A. K. Cheetham, Angew. Chemie - Int. Ed. 2011, 50, 3067.

[15] K. W. Chapman, G. J. Halder, P. J. Chupas, J. Am. Chem. Soc. 2009, 131, 17546.

[16] T. D. Bennett, J. C. Tan, Y. Yue, E. Baxter, C. Ducati, N. J. Terrill, H. H. M. Yeung, Z. Zhou, W. Chen, S. Henke, A. K. Cheetham, G. N. Greaves, Nat. Commun. 2015, 6, 1.

[17] T. D. Bennett, Y. Yue, P. Li, A. Qiao, H. Tao, N. G. Greaves, T. Richards, G. I. Lampronti, S. A. T. Redfern, F. Blanc, O. K. Farha, J. T. Hupp, A. K. Cheetham, D. A. Keen, J. Am. Chem. Soc. 2016, 138, 3484. 
[18] R. S. K. Madsen, A. Qiao, J. Sen, I. Hung, K. Chen, Z. Gan, S. Sen, Y. Yue, Science 2020, 367, 1473.

[19] G. Kaur, R. K. Rai, D. Tyagi, X. Yao, P. Z. Li, X. C. Yang, Y. Zhao, Q. Xu, S. K. Singh, J. Mater. Chem. A 2016, 4, 14932.

[20] L. Frentzel-Beyme, M. Kloß, R. Pallach, S. Salamon, H. Moldenhauer, J. Landers, H. Wende, J. Debus, S. Henke, J. Mater. Chem. A 2019, 7, 985.

[21] L. Frentzel-Beyme, M. Kloß, P. Kolodzeiski, R. Pallach, S. Henke, J. Am. Chem. Soc. 2019, 141, 12362.

[22] R. Banerjee, A. Phan, B. Wang, C. Knobler, H. Furukawa, M. O’Keeffe, O. M. Yaghi, Science 2008, 319, 939.

[23] Q. Zheng, Y. Zhang, M. Montazerian, O. Gulbiten, J. C. Mauro, E. D. Zanotto, Y. Yue, Chem. Rev. 2019, 119, 7848.

[24] A. Qiao, T. D. Bennett, H. Tao, A. A. Krajnc, G. Mali, C. M. Doherty, A. W. Thornton, J. C. Mauro, G. N. Greaves, Y. Yue, Sci. Adv. 2018, 4, eaao6827.

[25] A. Schneemann, V. Bon, I. Schwedler, I. Senkovska, S. Kaskel, R. A. Fischer, Chem. Soc. Rev. 2014, 43, 6062 .

[26] S. Gadipelli, W. Travis, W. Zhou, Z. Guo, Energy Environ. Sci. 2014, 7, 2232.

[27] C. P. Wong, P. J. Miller, J. Energ. Mater. 2005, 23, 169.

[28] S. Yang, R. Lv, C. Wang, Y. Liu, Z. Song, J. Alloys Compd. 2013, 579, 628.

[29] C. Hu, Y. C. Huang, A. L. Chang, M. Nomura, J. Colloid Interface Sci. 2019, 553, 372.

[30] C. Li, X. Lou, Q. Yang, Y. Zou, B. Hu, Chem. Eng. J. 2017, 326, 1000.

[31] X.-L. Wu, Y.-G. Guo, J. Su, J.-W. Xiong, Y.-L. Zhang, L.-J. Wan, Adv. Energy Mater. 2013, 3, 1155 .

[32] M. Gustafsson, X. Zou, J. Porous Mater. 2013, 20, 55.

[33] M. Stepniewska, M. B. Østergaard, C. Zhou, Y. Yue, J. Non. Cryst. Solids 2020, 530, 119806. 
ToC figure

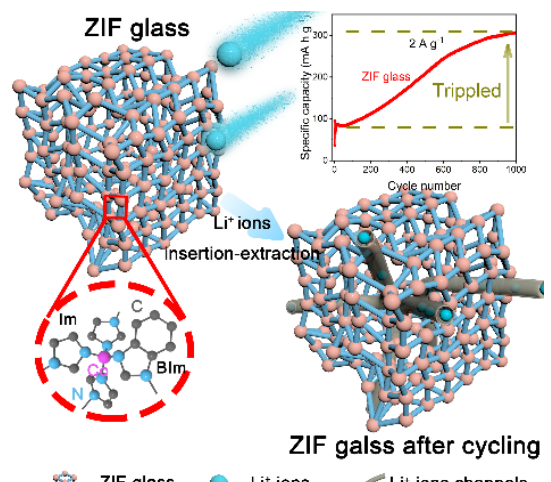

Metal-organic framework glass anode with an exceptional cycling-induced capacity enhancement for lithium ion batteries

Chengwei Gao, Zhenjing Jiang, Peixing Wang, Lars Rosgaard Jensen, Yanfei Zhang*, Yuanzheng Yue*

A ZIF glass (melt-quench Co ZIF-62 glass), for the first time, is evaluated as anode for high performance LIBs. This ZIF glass anode exhibits a striking capacity enhancement along with cycling. This exceptional phenomenon might be related to the unique structure of ZIF glass, i.e., high energy state, short range disorder. 


\section{Supporting Information}

\section{Metal-organic framework glass anode with an exceptional cycling-induced capacity enhancement for lithium ion batteries}

Chengwei Gao, Zhenjing Jiang, Peixing Wang, Lars Rosgaard Jensen, Yanfei Zhang*, Yuanzheng Yue*

C. Gao, Z. Jiang, P. Wang, Prof. Y. Zhang, Prof. Y. Yue

School of Materials Science and Engineering, Qilu University of Technology (Shandong Academy of Science), Jinan 250353, China

E-mail: zhang-yanfei@hotmail.com (Prof. Y.Zhang)

C. Gao, Prof. L.R. Jensen, Prof. Y. Yue

Department of Chemistry and Bioscience, Aalborg University, 9220 Aalborg, Denmark E-mail: yy@bio.aau.dk (Prof. Y. Yue)

Prof. L.R. Jensen

Department of Materials and Production, Aalborg University, 9220 Aalborg, Denmark

\section{Contents}

1. An enlarged view of Raman spectrum of ZIF crystal, ZIF glass and amorphous ZIF

2. Fourier-transform infrared spectra of ZIF crystal, ZIF glass and amorphous ZIF

3. $\mathrm{N}_{2}$ adsorption/desorption isothermal curves and pore size distribution of ZIF crystal, ZIF glass and amorphous ZIF

4. Voltage profiles and cycling performance of ZIF crystal, ZIF glass and amorphous ZIF

5. Cyclic voltammetry curves of ZIF crystal

6. Equivalent circuit model for fitting Nyquist plots of ZIF crystal, ZIF glass and amorphous ZIF

7. XPS spectra of ZIF crystal, ZIF glass and amorphous ZIF after cycling

8. XRD patterns of ZIF crystal, ZIF glass and amorphous ZIF after cycling 
1.

(a)

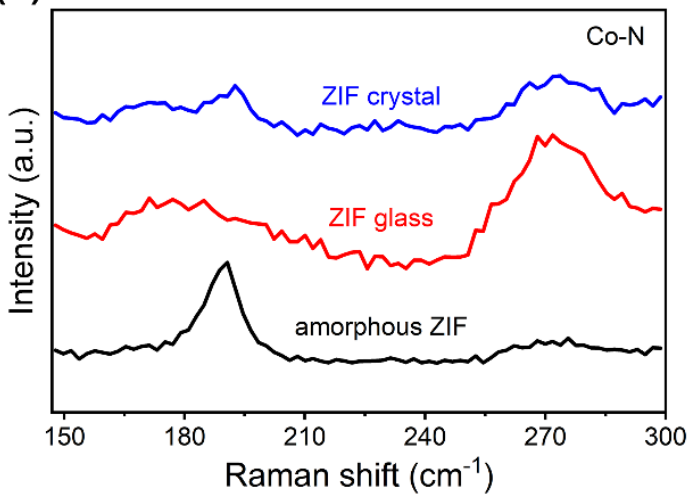

(b)

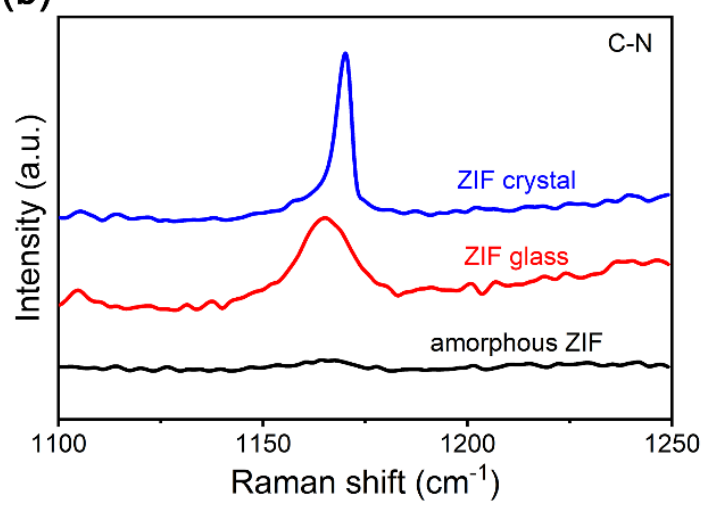

Figure S1. An enlarged view of Raman spectra of ZIF crystal, ZIF glass and amorphous ZIF within the frequency range of (a) $100-300 \mathrm{~cm}^{-1}$ and (b) $1100-1250 \mathrm{~cm}^{-1}$, respectively.

2.

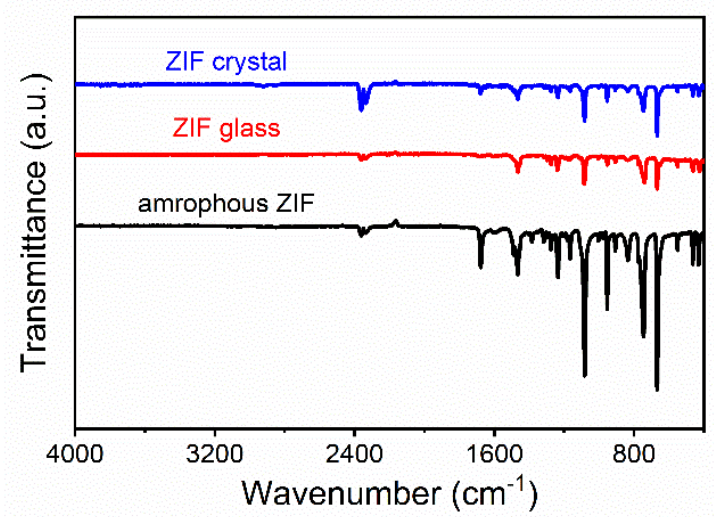

Figure S2. Fourier-transform infrared spectra of ZIF crystal, ZIF glass and amorphous ZIF, respectively. 
3.

(a)

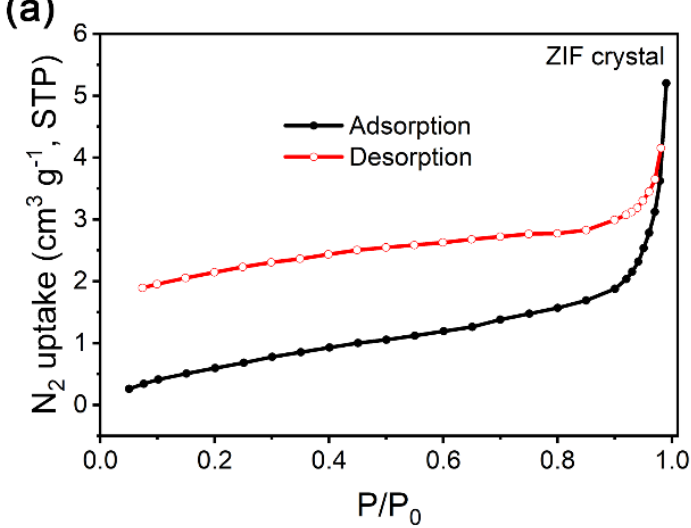

(c)

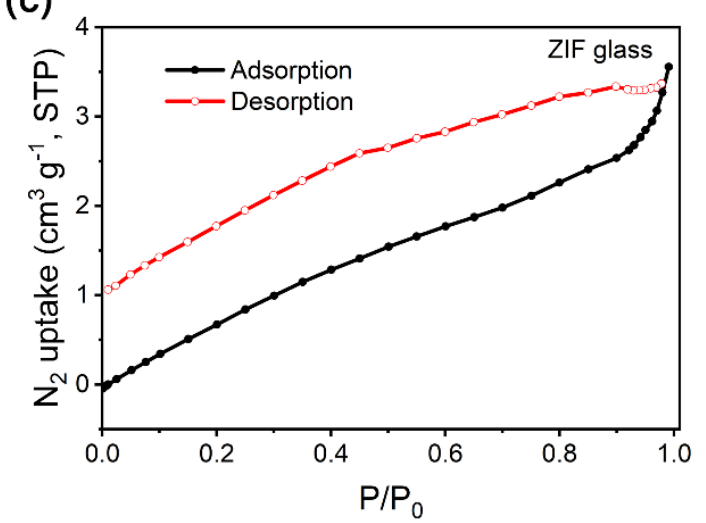

(e)

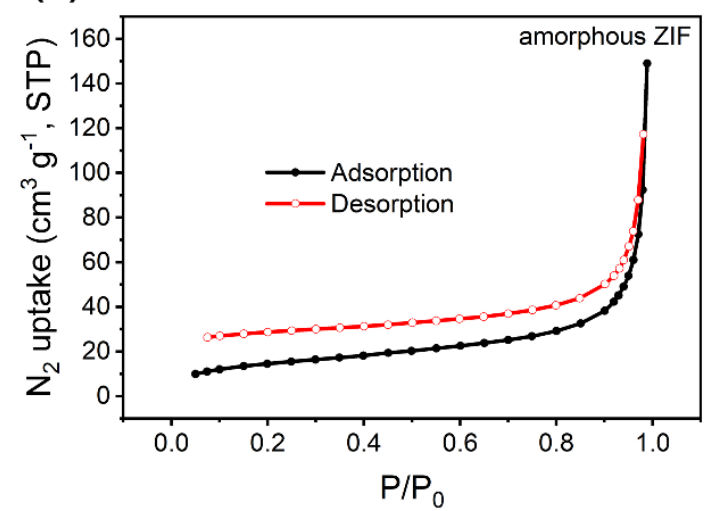

(b)

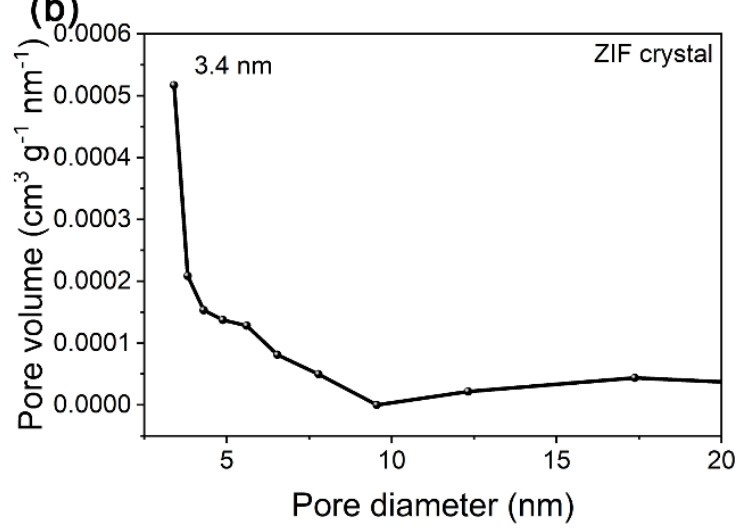

(d)

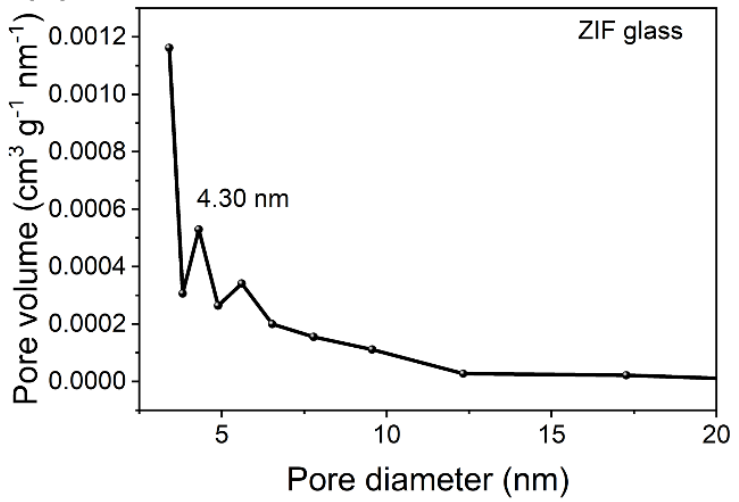

(f)

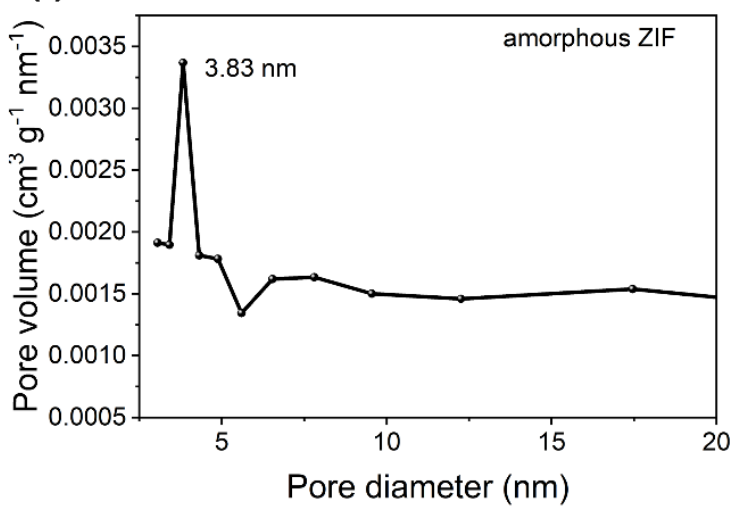

Figure S3. $\mathrm{N}_{2}$ adsorption/desorption isothermal curves and pore size distribution of ZIF crystal, ZIF glass and amorphous ZIF, respectively. 
4.

(a)

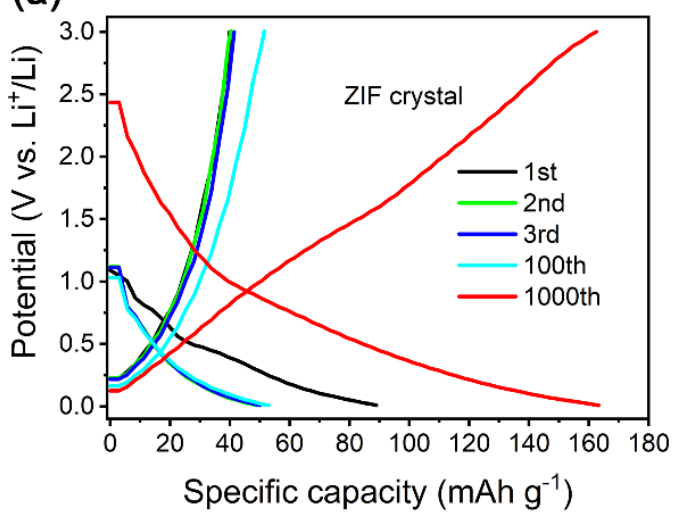

(c)

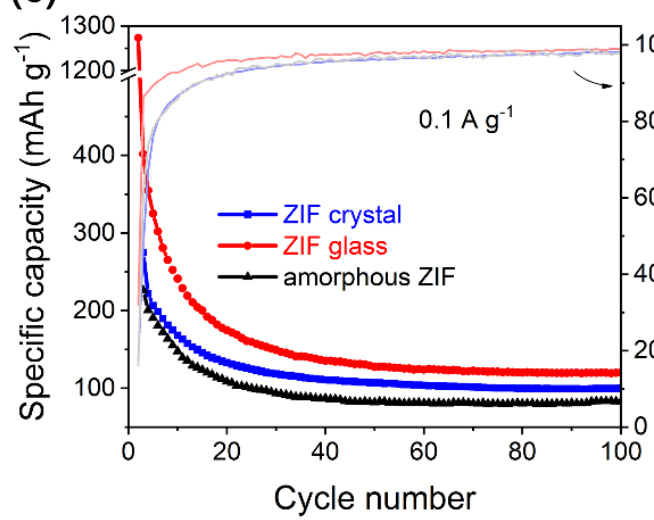

(e)

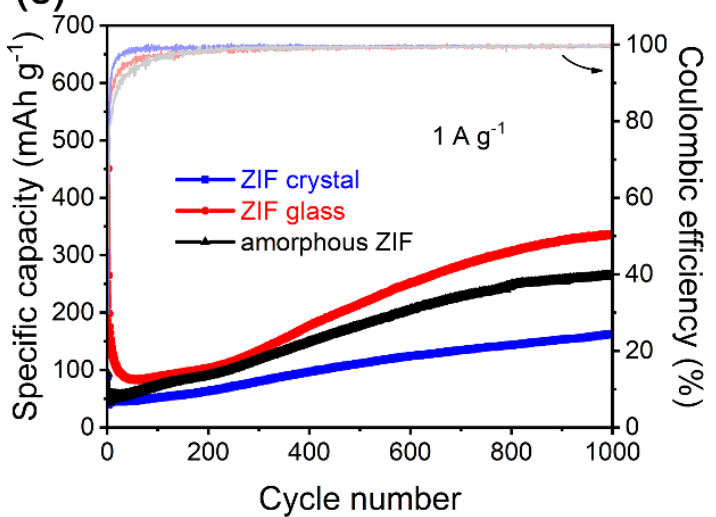

(b)

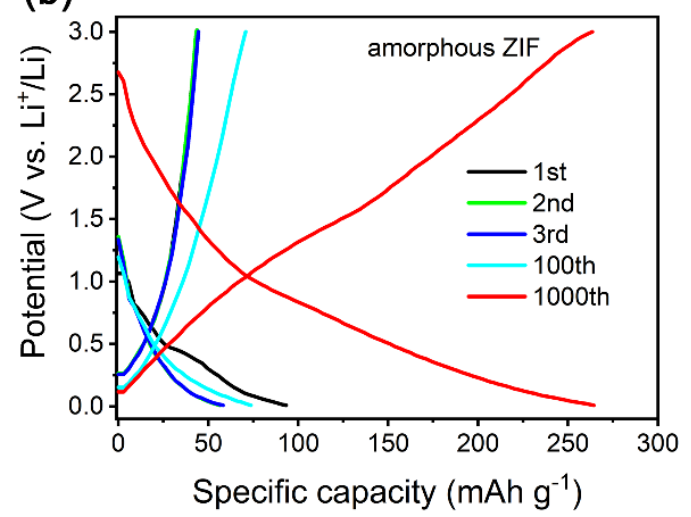

(d)

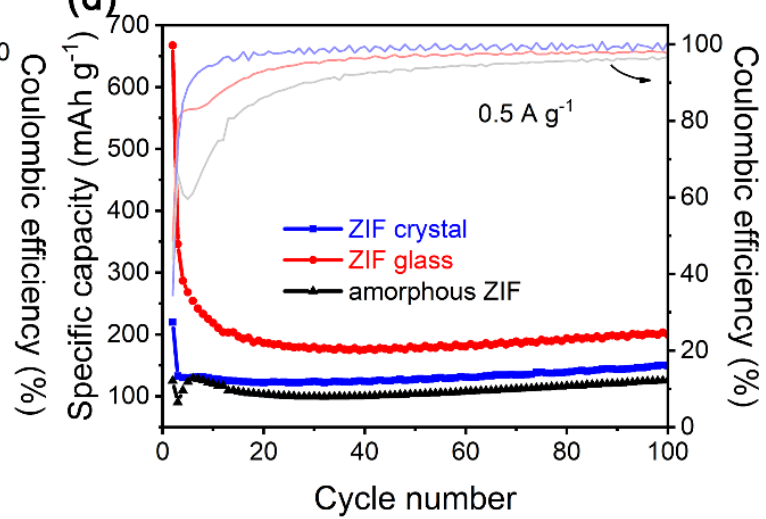

(f)

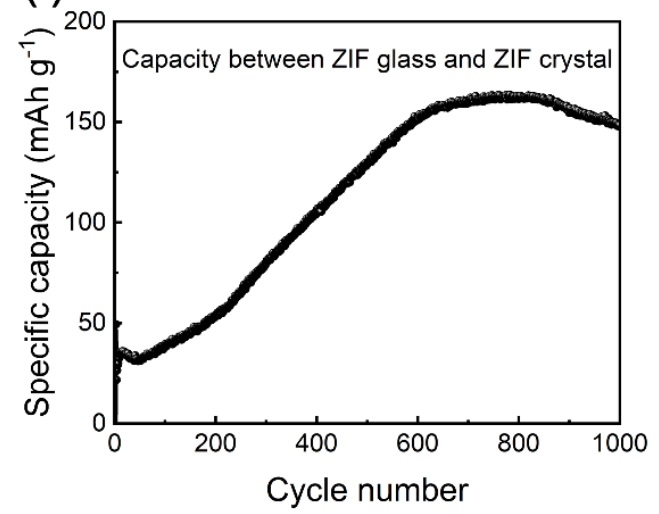

Figure S4. Galvanostatic charge-discharge voltage profiles of $1^{\text {st }}, 2^{\text {nd }}, 3^{\text {rd }}, 100^{\text {th }}$ and $1000^{\text {th }}$ cycles of (a) ZIF crystal and (b) amorphous ZIF at a current density of $1 \mathrm{~A} \mathrm{~g}^{-1}$. Cycling performance at a current density of $0.1 \mathrm{~A} \mathrm{~g}^{-1}$ (c) and $0.5 \mathrm{~A} \mathrm{~g} \mathrm{~g}^{-1}$ (d) for 100 cycles and $1 \mathrm{~A} \mathrm{~g}^{-1}$ for 1000 cycles (e) of ZIF crystal, ZIF glass and amorphous ZIF. (f) Capacity difference between ZIF glass and ZIF crystal at a current density of $2 \mathrm{~A} \mathrm{~g}^{-1}$. 
5.

(a)

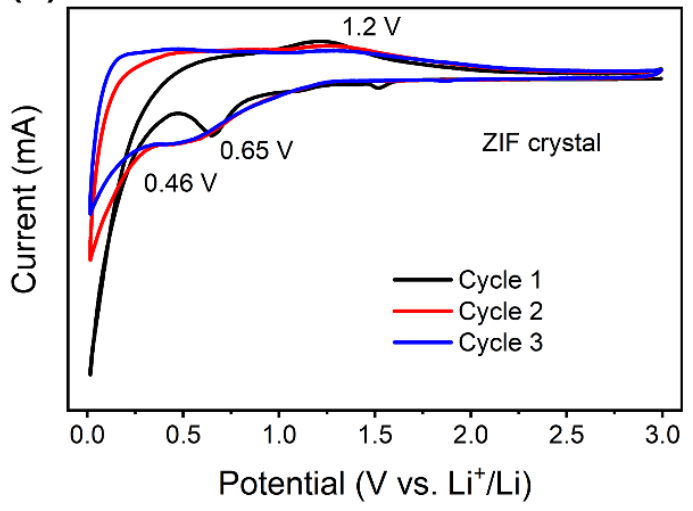

(b)

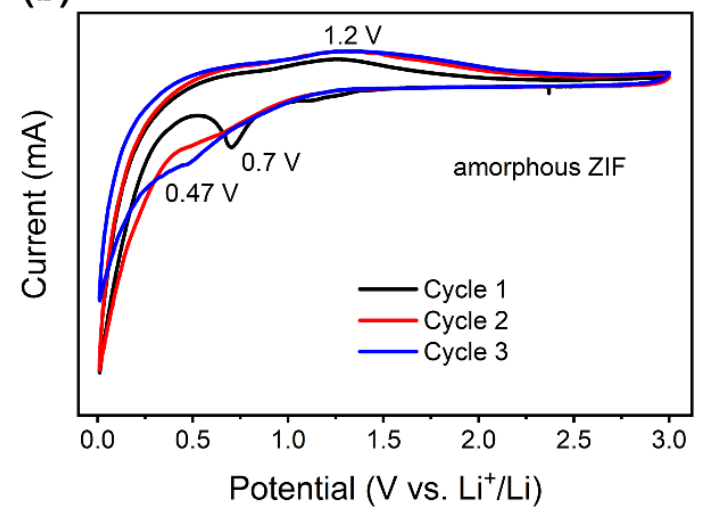

Figure S5. Cyclic Voltammetry curves of (a) ZIF crystal and (b) amorphous ZIF within the range from 0.01 to $3.0 \mathrm{~V}$ at a scan rate of $0.1 \mathrm{mV} \mathrm{s}^{-1}$.

6.

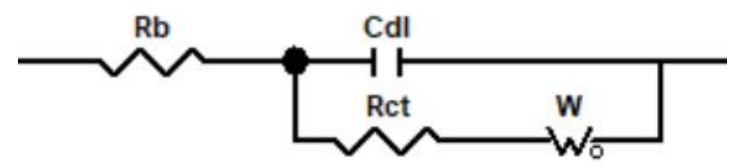

Figure S6. Equivalent circuit model for fitting Nyquist plots of ZIF crystal, ZIF glass and amorphous ZIF.

\begin{tabular}{|l|l|l|l|}
\hline & $\operatorname{Re}(\Omega)$ & $\operatorname{Rct}(\Omega)$ & $\mathrm{C}(\mathrm{F})$ \\
\hline ZIF crystal & 2.68 & 258 & 0.72 \\
\hline ZIF glass & 4.95 & 218 & 0.64 \\
\hline Amorphous ZIF & 3.57 & 52 & 0.79 \\
\hline
\end{tabular}

Table S1. Calculated values of components from EIS equivalent circuit model. 
7.

(a)

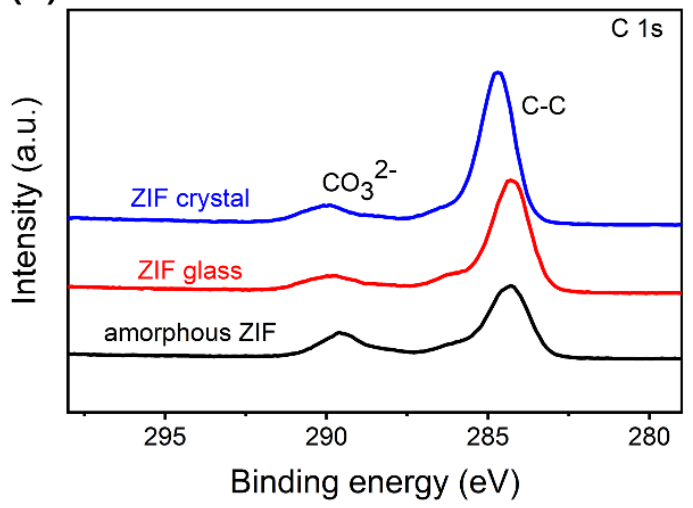

(b)

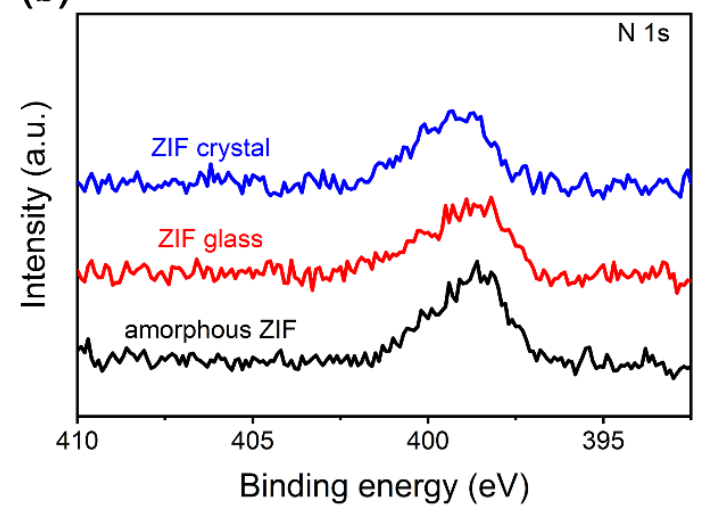

Figure S7. XPS high-resolution spectra of C 1s (a) and N 1s (b) of ZIF crystal, ZIF glass and amorphous ZIF after1000 charge-discharge cycles.

8.

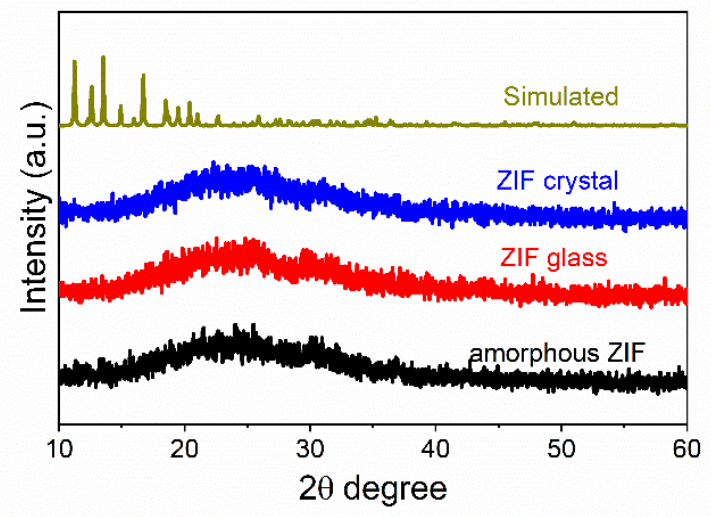

Figure S8. Ex-situ XRD patterns obtained from 10 to 60 degree of ZIF crystal, ZIF glass and amorphous ZIF after cycling. 